\title{
Spectral Polarimetric Method for Turbulence Intensity Estimation in Rain
}

\author{
Anna N. Rudiakova, Daria N. Turenko, and Felix J. Yanovsky \\ Electronics Department \\ National Aviation University (NAU) \\ Kiev, Ukraine \\ yanovsky@nau.edu.ua
}

\begin{abstract}
In this paper we develop an algorithm for turbulence intensity estimation based on the spectral polarimetric approach to the remote sensing of rain. The algorithm is compared with traditional Doppler spectrum width estimation; advantages and disadvantages of the two are analyzed, and recommendations for further research and practical applications are made
\end{abstract}

Keywords-Doppler radar; polarimetric padar; remote sensing; meteorological radar; turbulence intensity retrieval; signal simulation; signal processing

\section{INTRODUCTION}

Turbulence intensity remote estimation with help of radar is normally based on measuring Doppler spectrum parameters like Doppler spectrum width (DSW) or mean Doppler velocity (MDV) [1]. The accuracy of such estimates is limited because both DSW and MDV of the reflected signal depend not only on the turbulence intensity. Some parameters of radar, conditions of observation, features of objects, and other factors like limited samples influence on the results of measurements. This is a reason of the fact that relationships between measurable variables and wanted parameters of turbulence are of statistical nature. Another essential factor, which underlines the statistical nature of this task, is a receiver noise. However, it is important to understand that even in case of high signal-to-noise ratio the task of turbulence intensity retrieval from the reflected radar signal is a very complicated inverse problem which strictly speaking does not have a unique solution. We are dealing with indirect measurements when we can measure some radar signal parameters (measurable variables) which are statistically related with wanted parameter(s) of the object of the remote observation. In such cases it is preferable to have a set of measurable variables, and the result of joint usage of this set will be better if measurable variables are independent one from another.

Polarimetric parameters which are associated with the shape of the scatterer [2] are independent on Doppler parameters associated with velocity of the scatterer. However, a conventional opinion existed that polarimetric parameters are not applicable for turbulence estimation because they do not carry any useful information about turbulence.
In [3], [4], [5] spectral-polarimetric parameters were introduced, and it was shown that, for example, spectral differential reflectivity essentially depends on turbulence intensity in the resolution volume filled with scatterers, in particular, water drops. Another approach to associate the radar polarimetry with wind conditions (and turbulence) is based on the supposition that air flow directly influences onto the shape of a water droplet deforming it that leads to change polarimetric parameters [6], [7], [8].

Application of spectral-polarimetric parameters as measurable variables in the task of turbulence estimation is very attractive [9]. However, known algorithms used to estimate such parameters are not applicable for real time measurements because they need data averaging during rather long time [10].

In this paper we propose advanced models for spectralpolarimetric parameters and develop a new simplified algorithm which can be implemented for operational meteorological radar system.

\section{Spectral-Polarimetric Remote Sensing of Clouds AND PRECIPITATION}

\section{A. Doppler-Polarimetric Radar}

A Doppler-polarimetric radar is a coherent radar system which is also capable to control polarization of sounding waveform and to receive two orthogonal polarization component of the reflected signal. Modern meteorological radars can be pulse-Doppler systems or continuous wave (CW) systems with different types of modulation, for example FM CW radar. In this paper we develop an advanced model that actually can be adjusted to any Doppler-polarimetric radar (pulse or $\mathrm{CW}$ ) that is able to produce reflected signal at different combinations of polarization components for transmitted and received electromagnetic wave, for example, $h h, v v, h v$, and $v h$ with $h$ and $v$ as horizontal and vertical linear polarization correspondingly, and the first sign in the pair means polarization of a received component, while the second sign means polarization of the sounding waveform. In addition to the intensity of the precipitation, a Doppler-polarimetric radar is capable of detecting the motion of scatterers (Doppler) and is potentially sensitive to the shape of scatterers (polarimetric). Both types of Doppler-polarimetric data (pulse 
and $\mathrm{CW}$ ) can be analyzed to determine the structure of storms and their potential to cause severe weather.

\section{B. Spectral Differential Reflectivity (SDR)}

In radar meteorology the traditional differential reflectivity $Z d r$ [1], [2], [5] is defined as

$$
\begin{aligned}
& Z d r=10 \lg \left(Z_{h h} / Z_{v v}\right)=10 \lg \left(P_{h h} / P_{v v}\right)= \\
& 10 \lg \left(\sigma_{h h} / \sigma_{v v}\right)=10 \lg <\left|s_{h h}\right|^{2}>/<\left|s_{v v}\right|^{2}>
\end{aligned}
$$

where $Z$ means radar reflectivity factor, $P$ is average received power, $\sigma$ is RCS, and $\left\langle|s|^{2}>\right.$ is an element of the covariance matrix [1], [5]; the indexes $h$ and $v$ have been explained above in subsection $A$. The elements of covariance matrix $<\left|s_{h h}\right|^{2}>$ and $\left\langle\left|s_{v v}\right|^{2}\right\rangle$ are actually second-order moments (covariations) $R_{h h}$ and $R_{v v}$ of the random processes $s_{h h}$ and $s_{v v}$, which are principal components of the scattering matrix.

In contrast to (1), SDR $s Z d r(v)$ is introduced as $Z d r$ for each velocity bin, that is, as a function of Doppler velocity or frequency. Let us suppose that Doppler spectra at orthogonal polarizations $S_{h h}(v)$ and $S_{v v}(v)$ are known. Based on these spectra, SDR or $s Z d r(v)$ is defined as

$$
s Z d r(v)=10 \log \left[S_{h h}(v) / S_{v v}(v)\right] .
$$

A conventional polarimetric parameter like $Z d r(1)$ is a number, which integrally describes some features of the entire resolution volume of meteorological object under observation in given time. A spectral-polarimetric characteristic like SDR $s Z d r(v)$ contains some information on the fine structure of the object within the resolution volume. More exactly, it provides a kind of super-resolution because allows to estimate polarization properties of different elements within the resolution volume, for example, droplets which are moving with different velocities though without specifying the exact location of them inside the resolution volume.

\section{Slope of SDR}

In special case of rain observation, SDR theoretically is a monotonously increasing curve at least within the main part of the Doppler spectrum [5]. This is caused by three reasons: 1) the bigger a droplet the faster it is falling down in the air (Stokes law); 2) the bigger droplets are more oblate, that is, more different from spherical shape; 3) when falling down, a spheroidal (or even ellipsoidal) droplet is oriented almost horizontally, that is, its horizontal size is more than vertical one.

A phenomenological model that relates parameters of rain microstructure, turbulence, radar systems parameters, and conditions of observation was developed [4], [11]. The model took into account inertial properties of raindrops according to [12]. It was shown analytically and by simulation [3], [4], [5] that the steepness of SDR curve is of maximum in stagnant air when turbulence is absent. Increased turbulence intensity leads to the fact that the SDR curve becomes less steep, that is, its slope decreases. The slope of $s Z d r(v)$ (SLP) in the form of slope ratio of the tangent at the inflection point of the curve was introduced [10], [13] as SDR-curve the parameter.

\section{RELATIONSHIP BETWEEN TURBULENCE INTENSITY IN RAIN AND SPECTRAL DIFFERENTIAL REFLECTIVITY}

Here we describe the advanced model, which allows calculating spectral polarimetric parameters of reflected radar signal depending on meteorological object parameters, and provide investigation of the relationship between turbulence intensity in rain and spectral-polarimetric parameters on the basis of the developed model.

\section{A. Advanced Phenomenological Model}

Using the initial phenomenological model developed in [4], [11] with slightly different approach concerning the incident wave polarization, the advanced phenomenological model was built. It allows to simulate the real-time spectrum data for coand cross-polarized components depending on several meteorological and radar sounding wave parameters, such as eddy dissipation rate, median drop diameter, elevation angle, etc. Therefore, it is very helpful for theoretical investigation of the turbulence influence on the spectral-polarimetric parameters.

\section{B. Results of Calculations and Simulation}

The modeling results are shown in Figs. 1-6 for the median drop diameter $D_{0}=1.1 \mathrm{~mm}$, drop size distribution parameter $\mu=5$, dielectric permittivity of water $\varepsilon_{r}=81$, radar radial resolution of $3 \mathrm{~m}$. The varying parameters are antenna elevation angle and eddy dissipation rate (EDR).

As it was expected from theory, there is zeroth $s Z d r$ correspond to zenith antenna direction (antenna elevation angle equal to $90^{\circ}$ ). So, in order to successful use of proposed algorithm for turbulence intensity estimation, one need to direct an antenna far below then zenith.

\section{Co-polarized spectral components Shh (red), Svv (blue)}

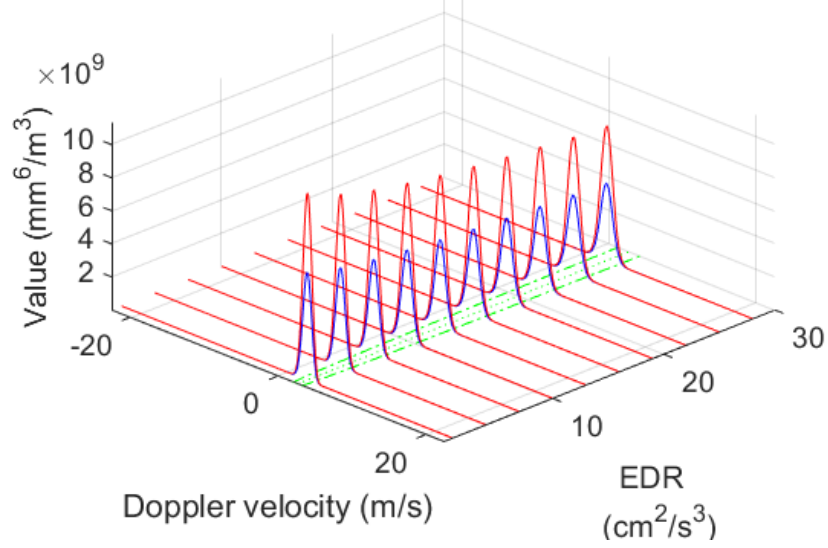

Fig. 1. Simulated Doppler spectra for two orthogonal polarizations $S_{h h}(v)$ and $S_{v v}(v)$ that correspond to the antenna elevation angle $30^{\circ}$. Green lines show the median doppler velocity (dotted) and doppler spactra width (dash and dotted) values according to the eddy dissipation rate 


\section{Spectral differential reflectivity sZdr (red) and slope line of sZdr (blue)}

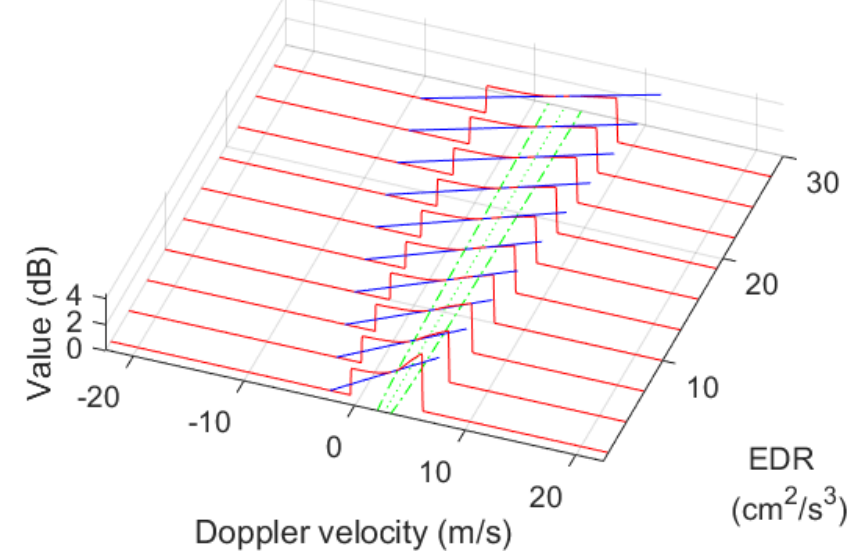

Fig. 2. Calculated spectral differential reflectivity and corresponding slope lines for the antenna elevation angle $30^{\circ}$. Green lines show the median doppler velocity (dotted) and doppler spactra width (dash and dotted) values according to the eddy dissipation rate

\section{Co-polarized spectral components Shh (red), Svv (blue)}

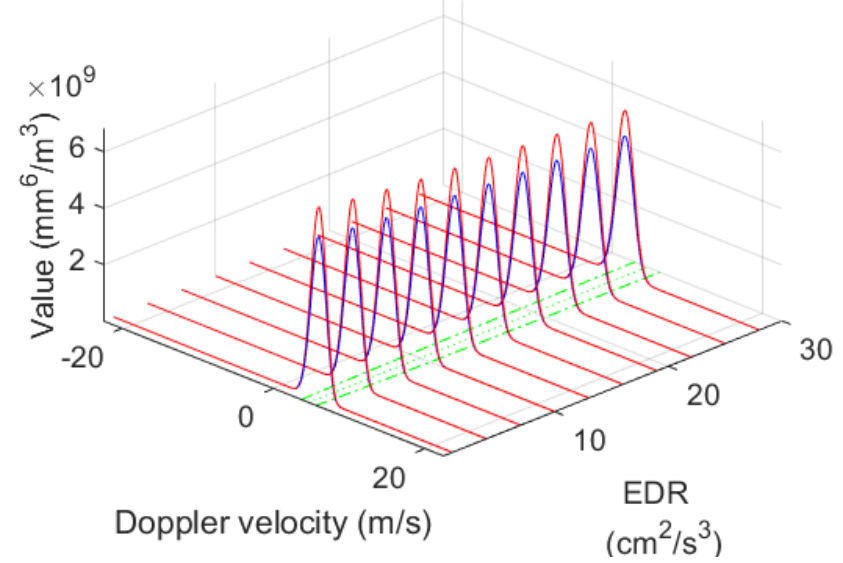

Fig. 3. Simulated Doppler spectra for two orthogonal polarizations $S_{h h}(v)$ and $S_{v v}(v)$ that correspond to the antenna elevation angle $60^{\circ}$. Green lines show the median doppler velocity (dotted) and doppler spactra width (dash and dotted) values according to the eddy dissipation rate

Figs. 1 and 3 show the simulated Doppler spectra components $S_{h h}(v)$ and $S_{v v}(v)$ for the antenna elevation angles of $30^{\circ}$ and $60^{\circ}$, respectively. As can be seen, the vertical polarization component approaches horizontal one with elevation angle increasing. It is also clear, that Doppler spectrum width (green dash-and-dotted lines in Figs.) become wider with eddy dissipation rate increasing for each of considered elevation angles.

Figs. 2 and 4 show the calculated spectral differential reflectivity $s Z d r(v)$ and SLP lines for the same antenna elevation angles, as Figs. 1, 3. It should be noted, that maximum values of sZdr become decreasing with elevation angle or eddy dissipation rate increasing.

\section{Spectral differential reflectivity sZdr (red) and slope line of sZdr (blue)}

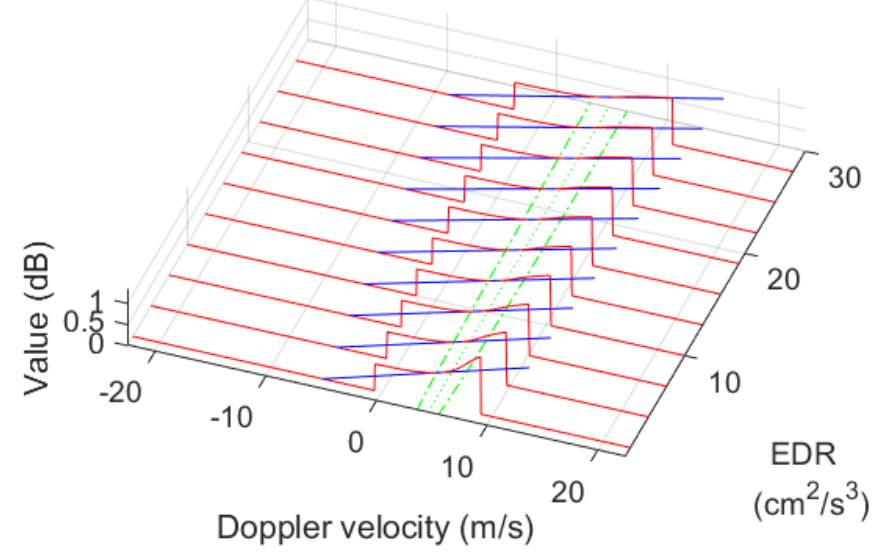

Fig. 4. Calculated spectral differential reflectivity and corresponding slope lines for the antenna elevation angle $60^{\circ}$. Green lines show the median doppler velocity (dotted) and doppler spactra width (dash and dotted) values according to the eddy dissipation rate

\section{Co-polarized spectral components Shh (red), Svv (blue)}

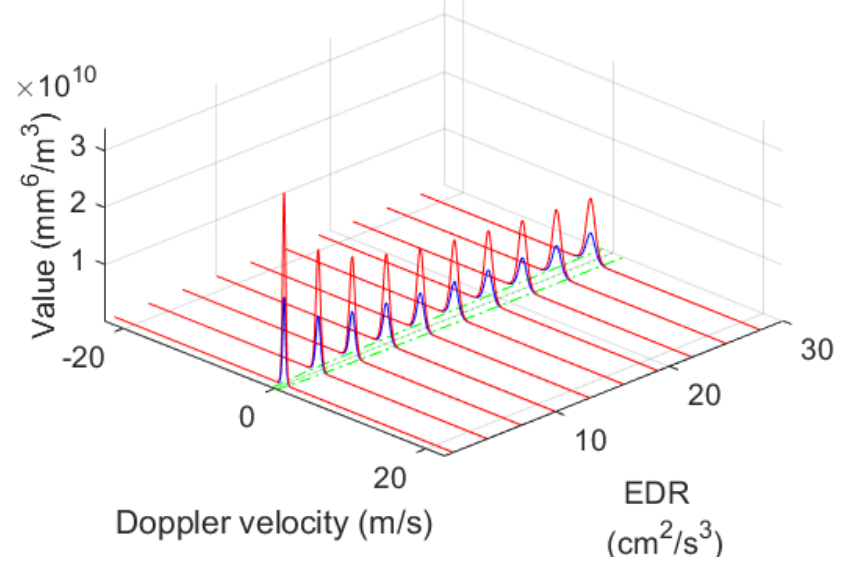

Fig. 5. Simulated Doppler spectra for two orthogonal polarizations $S_{h h}(v)$ and $S_{v v}(v)$ that correspond to the antenna elevation angle $0^{0}$. Green lines show the median doppler velocity (dotted) and doppler spactra width (dash and dotted) values according to the eddy dissipation rate

Special case of horizontal sounding (antenna elevation angle $0^{0}$ ) is shown in Figs. 5, 6. In the absence of drop falling velocity radial projection, the Doppler spectrum width is defined mainly by turbulence in the presenting model. The sZdr becomes the constant value outside the Doppler spectrum width region around the mean Doppler velocity. In order to acquire the SLP in such a case, it should be calculated using the only a few samples of sZdr located in between the mean Doppler velocity value and the same value plus Doppler spectrum width. This approach is applicable also for the highest elevation angles and was used to build SLP lines shown in Figs. 2, 4. Using the only few points of sZdr instead whole samples set for estimation of SLP improves the calculation speed and can be utilized in operational algorithm. 


\section{Spectral differential reflectivity sZdr (red) and slope line of sZdr (blue)}

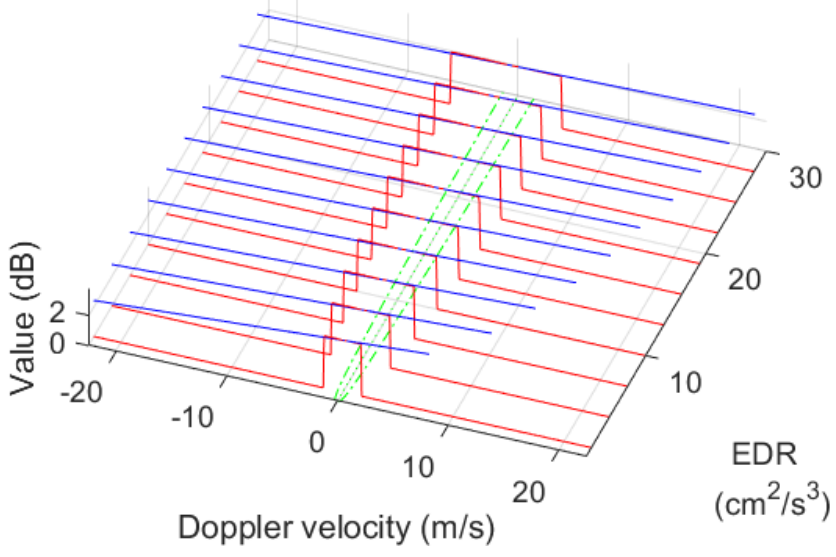

Fig. 6. Calculated spectral differential reflectivity and corresponding slope lines for the antenna elevation angle $0^{\circ}$. Green lines show the median doppler velocity (dotted) and doppler spactra width (dash and dotted) values according to the eddy dissipation rate

\section{SDR ALGORITHM FOR TURBULENCE INTENSITY ESTIMATION}

One of difficulties with SLP estimation using experimental data is the statistical nature of initial data sets. Therefore, some method of short time series spectral analysis should be implemented to smooth the spectrum oscillations.

\section{A. Data Descriptopn}

The experimental time series of orthogonally polarized radar signals acquired by PARSAX (Polarimetric Agile Radar in S- and X-band) [14] with elevation angle $5^{0}$. There are 512 time samples recorded for each range bin with $1 \mathrm{~ms}$ step. Fig. 7 shows the mean Doppler velocities map for consideration region with rain.

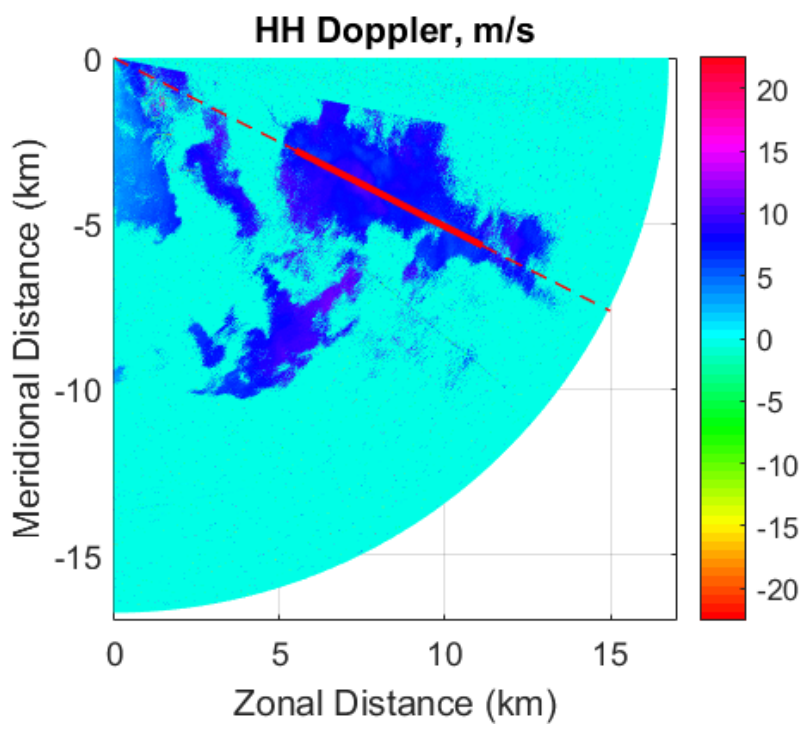

Fig. 7. Mean Doppler velocities map for the consideration region. Red line shows the antena azimuth angle used in calculations. Bold sector of this line correspond to the rainy zone

\section{B. Synthesis of Signal Processing Algorithm}

The operational algorithm of SLP estimation we propose consists of the following steps:

1) clutter reduction:

- performing fast Fourier transform of initial time series;

- removing spectral components around zero-Doppler frequency;

- performing inverse fast Fourier transform;

2) spectrum smoothing:

- spectrum estimation using Blackman-Tukey method;

- noise level reduction;

3) calculating sZdr and SLP as well as mean Doppler velocity, Doppler spectrum width and other parameters for processed spectra.

\section{Data Processing}

The first step of operational data processing algorithm is illustrated by Figs. 8, 9 for the range bin that correspond to $8 \mathrm{~km}$ radial distance from radar. Fig. 8 shows the initial time series for both vertically and horizontally polarized signals as well as the results of fast Fourier transform (FFT). The mature clutter signal can be seen at close to zero Doppler frequencies, that does not directly relate to the precipitations, but can strongly impact on rain spectrum estimation. Processed with zeroth Doppler filter time series and spectra of orthogonal polarizations $\mathrm{HH}$ and VV are shown in Fig. 9.

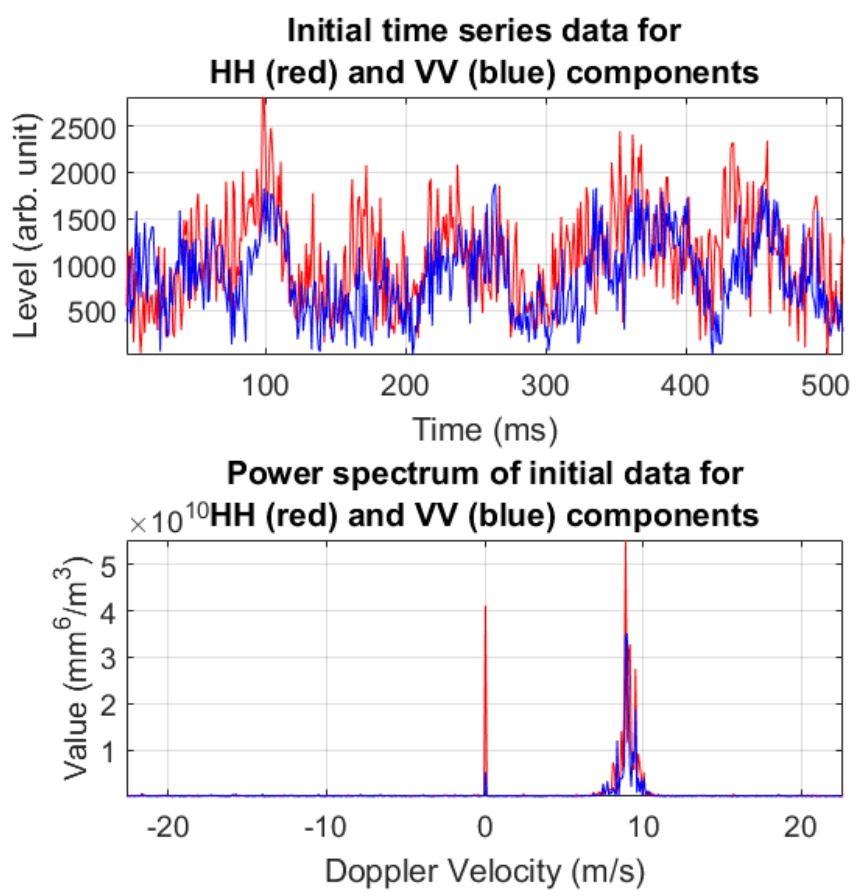

Fig. 8. Raw data for the two orthogonal polarizations HH and VV (top picture); FFT of the raw time series (bottom picture) that correspond to $8 \mathrm{~km}$ distance from radar with rainy resolution volume 
Time series data after clutter suppression, HH (red) and VV (blue) components

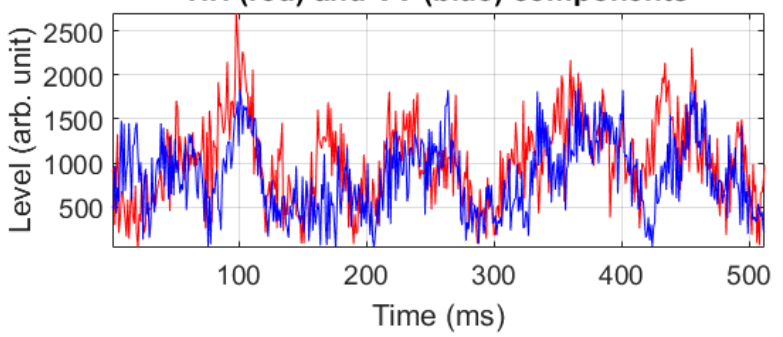

Power spectrum after clutter supression,

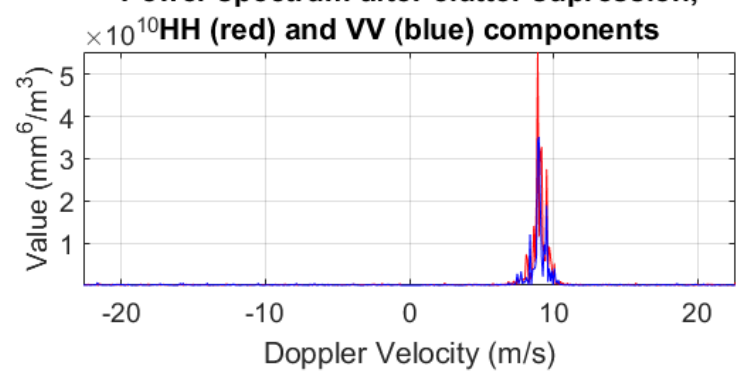

Fig. 9. Raw data for the two orthogonal polarizations $\mathrm{HH}$ and VV (top picture); FFT of the raw time series (bottom picture) that correspond to $8 \mathrm{~km}$ distance from radar with rainy resolution volume.

The second step of proposing algorithm concerns the spectrum smoothing using the Blackman and Tukey method. According to the number of samples in time series, there was 50-samples Hann window selected for auto-covariation function [15]. Processing results before and after noise level reduction are shown in Fig. 10.

Third algorithm's step allows to calculate sZdr, SLP and other parameters. Fig. 11 shows the sZdr within processing region that correspond to the interval around mean Doppler frequency. The SLP line is also depicted.

\section{Smoothed spectra for} HH (red) and VV (blue) components

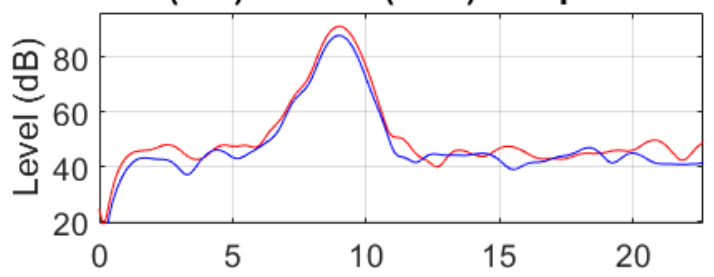

\section{Smoothed spectra after noise level reduction $\mathrm{HH}$ (red) and VV (blue) components}

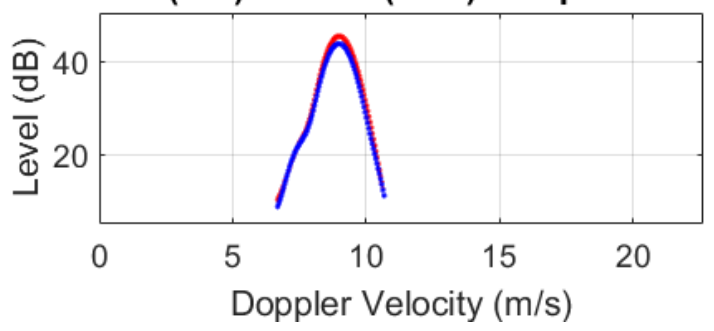

Fig. 10. Processed with Blackman-Tukey method spectra before (top picture) and after (buttom picture) noise level reduction

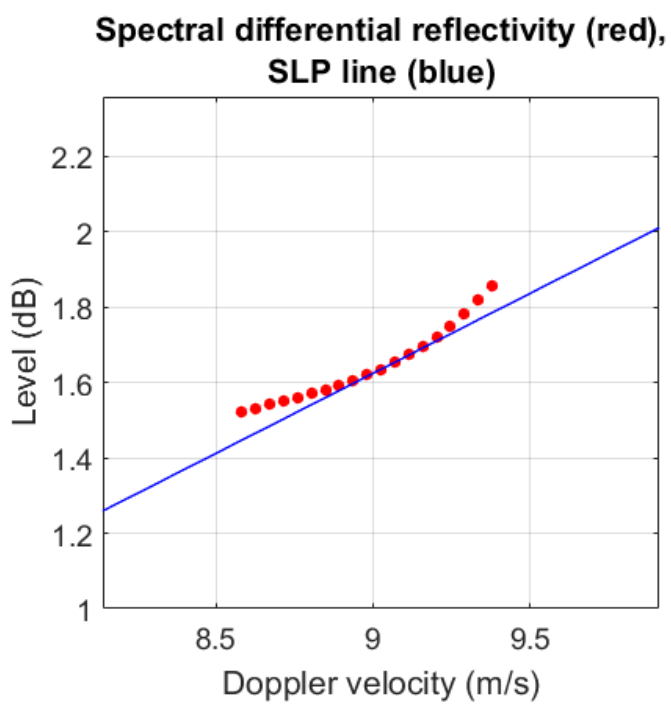

Fig. 11. Calculated sZdr and SLP line $(\operatorname{SLP}=0.42)$

Calculated parameters that correspond to the smoothed spectra are following: mean Doppler velocity $\mathrm{MDV}=8.9 \mathrm{~m} / \mathrm{s}$, Doppler spectrum width DSW $=0.43 \mathrm{~m} / \mathrm{s}$, eddy dissipation rate $\mathrm{EDR}=1.2 \mathrm{~cm}^{2} / \mathrm{s}^{3}$, median drop diameter $D_{0}=1.66 \mathrm{~mm}$, rainfall rate $R=21.4 \mathrm{~mm} / \mathrm{h}$.

\section{COMPARISON OF SDR AND DSW ALGORITHMS}

As it can be seen from Section III-B results, there is obvious that exist some relation between the slope of sZdr and eddy dissipation rate for elevation angles below the zenith. Fig. 12 shows the comparison of SLP and DSW dependences on eddy dissipation rate for the antenna elevation angle $30^{\circ}$.
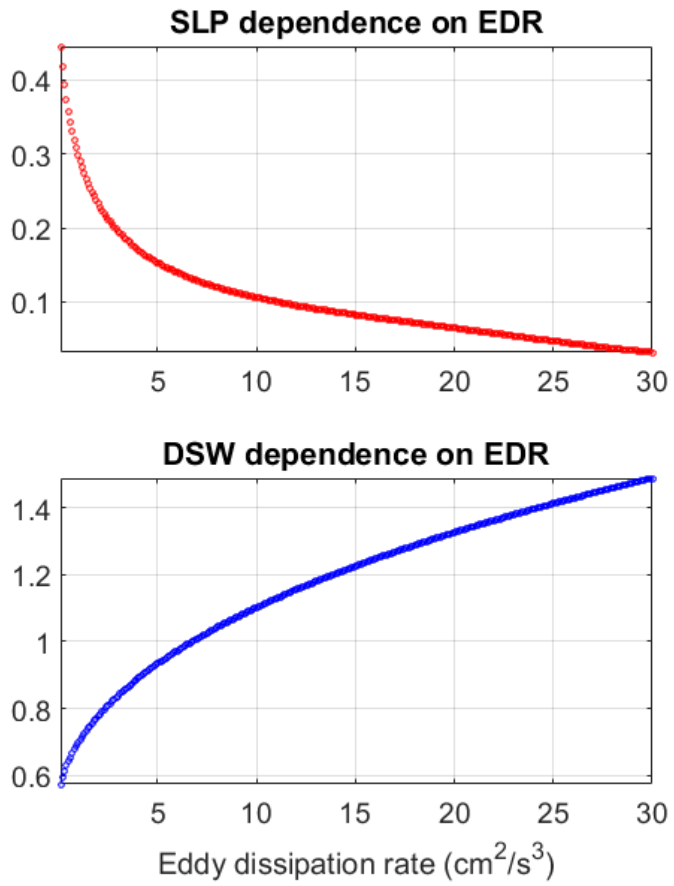

Fig. 12. Slope of sZdr (top picture) and Doppler spectrum width (bototm picture) dependencies on the eddy dissipation rate 


\section{CONCLUSION}

The simplified algorithm, which has been developed in this paper, can help in operational evaluation not only the slope of spectral differential reflectivity, but also other signal parameters of meteorological radar system, for example, Doppler spectrum width. However, the developed algorithm still needs additional experimental approbations in order to become widely used. Also, this approach is worthy to be deeper analyzed theoretically. This is planned to be done in future work.

\section{ACKNOWLEDGMENT}

The authors are grateful to the Delft University of Technology (TU-Delft), the Netherlands, and personally Prof. Alex Yarovoy and Dr. Oleg Krasnov for providing the measurement raw data.

\section{REFERENCES}

[1] R. Doviak and D. Zrnić, Doppler Radar and Weather Observations, San Diego: Academic Press, 1993.

[2] V. N. Bringi and V. Chandrasekar, Polarimetric Doppler Weather Radar: Principles and Applications, Cambridge, UK: Cambridge University Press, 2001.

[3] C. M. H. Unal, D. N. Moisseev, F. J. Yanovsky, and H. W. J. Russchenberg, "Radar Doppler polarimetry applied to precipitation measurements: introduction of the spectral differential reflectivity." Proceedings of the 30th International Conference on Radar Meteorology (AMS), Boston, 2001, pp. 316-318.

[4] Yanovsky, F. J., Model of the relationship between Doppler and polarization parameters of the radar signal from precipitation. Report No. IRCTR-S-002-98, Technical University, Delft, 1998, 108 pp.

[5] F. J. Yanovsky, "Inferring microstructure and turbulence properties in rain through observations and simulations of signal spectra measured with Doppler-polarimetric radars." In: Polarimetric Detection, Characterization, and Remote Sensing, M.I. Mishchenko et al. (eds.), NATO Science for Peace and Security Series C: Environmental Security, Springer Science+Business Media B.V. 2011, pp. 501-542.
[6] Yu. Averyanova, A. Averyanov, and F. J. Yanovsky, "The approach to estimating critical wind speed in liquid precipitation using radar polarimetry," 2012 Int. Conf. Mathematical Methods in Electromagnetic Theory (MMET), Kharkov, Ukraine, 2012, pp. 517-520.

[7] Yu. Averyanova, A. Averyanov, and F. J. Yanovsky, "Polarization signal components estimate in weather radar," Mathematical Methods in Electromagnetic Theory (MMET), Conference Proceedings, Odessa, Ukraine, 2008, pp. 360-362.

[8] Yu. Averyanova, A. Averjanov, and F. J. Yanovsky, "Doppler Polarization Radar Methods for Meteorological Applications," IEEE A\&E SYSTEMS Magazine, July 2014, Vol.29, No 7, pp. 64-73.

[9] Felix J. Yanovsky, "Spectral and polarimetric methods in active and passive remote sensing" (Invited paper), Proceedings, 2015 12th IEEE International Conference on Telecommunications in Modern Satellite, Cable, and Broadcasting Services (TELSIKS), Nis, Serbia, Oct 14-17, 2015, pp. 156-163.

[10] F. J. Yanovsky, C. M. H. Unal, and H. W. J. Russchenberg, Dopplerpolarimetric radar measurements of turbulence in rain. Report IRCTR-S006-03, Technical University, Delft, 2003, 102 pp.

[11] F. J. Yanovsky, "Phenomenological models of Doppler-polarimetric microwave remote sensing of clouds and precipitation", IEEE International Geoscience and Remote Sensing Symposium, IGARSS '02, Toronto, Canada, vol. 3, pp. 1905-1907, 2002.

[12] F. J. Yanovsky, "Simulation study of $10 \mathrm{GHz}$ radar backscattering from clouds and solution of the inverse problem of atmospheric turbulence measurements," Computational Electromagnetics, IEE CP 420, 1996, $188-193$.

[13] F.J. Yanovsky, H.W.J. Russchenberg, and C.M.H. Unal. "Retrieval of information about turbulence in rain by using Doppler-polarimetric radar," IEEE Transactions on Microwave Theory and Techniques, Feb., Vol. 53, No 2, 2005, pp. $444-450$.

[14] O.A. Krasnov, L.P. Ligthart, G.P. Babur, and F. van der Zwan, ThePARSAX - New Full Polarimetric FMCW Radar with DualOrthogonal Signals, Proceedings of the 8th International Symposium on Tropospheric Profiling, ISBN 978-90-6960-233-2, Delft, The Netherlands, October 2009. Editors, A. Apituley, H.W.J. Russchenberg, W.A.A. Monna. B 25, 2009, pp. S06-P08-1-S06-P08-4.

[15] R.B. Blackman, and Tukey, J.W., The measurement of power spectra, from the point of view of communications engineering, New York: Dover, 1959. 